\section{Concentrações de hemoglobina e ferritina sérica em escolares da rede pública municipal de Teresina, Piauí, Nordeste do Brasil}

\author{
Hemoglobin and serum ferritin \\ concentrations in public school children \\ from Teresina, in the State of Piauí, in \\ Northeast Brazil
}

Marize Melo dos Santos 1

Alcides da Silva Diniz 2

Nadir do Nascimento Nogueira 3
1,3 Departamento de Nutrição. Universidade Federal do Piauí. Campus Universitário Ministro Petronio Portela, Bloco 13 Teresina, PI, Brasil.CEP: 64.049-550.

E-mail: marizesantos@ufpi.br

2 Departamento de Nutrição. Universidade Federal de Pernambuco. Recife, PE, Brasil.

\begin{abstract}
Objectives: to assess iron nutritional status of public school children in Teresina, Piaui, Brazil.

Methods: a cross-sectional survey was conducted among 747 school children of both sexes, aged between seven and eleven years, who were randomly selected using a two-step sampling procedure, in August/September 2000. Children with hemoglobin (Hb) concentrations less than $11.5 \mathrm{~g} / \mathrm{dL}$ were evaluated as anemic and low body iron (Sfer $<15 \mu \mathrm{g} / L)$ was evaluated in 207 children.

Results: the prevalence of anemia was $14.3 \%$ (95\%CI 12.2-17.4) and of low body iron 20.3\% (95\%CI 15.2-26.6). Anemia and body iron depletion were not correlated with gender $(p=0.60 ; p=0.96$, respectively) or age group $(p=0.85 ; p=0.53$, respectively). SFer was not correlated $(r=0.1 ; p=0.168)$ with $\mathrm{Hb}$ concentrations. The prevalence of iron deficiency anemia $(\mathrm{Hb}<11.5 \mathrm{~g} / \mathrm{dL}$ and $\mathrm{SFer}<15.0 \mu \mathrm{g} / \mathrm{L})$ was $26.3 \%$ (95\% CI 17.3-37.5).

Conclusions: iron deficiency and anemia seem to be a public health problem among school children in Teresina. Concerted action to prevent and control these conditions is strongly recommended. However, all anemia cannot be explained by iron deficiency. Therefore, it may be wise to consider other etiologies, such as micronutrient status, parasite infestation, hereditary disorders and exposure to environmental pollutants.
\end{abstract}

Key words Hemoglobin, Ferritins, Schoolchildren, Iron deficiency, Anemia Iron-deficiency

\section{Resumo}

Objetivos: avaliar o estado nutricional de ferro em escolares de instituições públicas de ensino de Teresina, Piaui, Brasil.

Métodos: corte transversal, envolvendo amostra aleatória, selecionada em duas etapas, de 747 escolares (7-11 anos), de ambos os sexos, no período de agosto / setembro de 2000. A anemia foi rastreada em 747 escolares e para o diagnóstico adotou-se a concentração de hemoglobina $(\mathrm{Hb})<11,5 \mathrm{~g} / \mathrm{dL}$. As reservas corporais de ferro foram estimadas em 207 escolares, mediante análise das concentrações de ferritina sérica (FerS), considerando-se baixas reservas valores $<15 \mu \mathrm{g} / \mathrm{L}$.

Resultados: a prevalência de concentrações inadequadas de $\mathrm{Hb}(<11,5 \mathrm{~g} / \mathrm{dL})$ foi de $14,3 \%$ (IC95\% 12,2-17,4) e de reservas inadequadas de ferro (FerS $<$ $15,0 \mu \mathrm{g} / \mathrm{L})$ de 20,3\% (IC95\% 15,2-26,6). A prevalência de anemia foi semelhante entre os sexos $(p=0,60) \quad e$ as faixas etárias $(p=0,85)$. Comportamento distributivo similar foi observado no que diz respeito às reservas inadequadas de ferro, frentes às variáveis sexo $(p=0,19)$ e idade $(p=0,24)$. As concentrações de FerS não mostraram correlação $(r=0,1 ; p=0,168)$ com as de Hb. A prevalência de anemia ferropênica $(\mathrm{Hb}<11,5 \mathrm{~g} / \mathrm{dL}$ e FerS $<15,0$ $\mu \mathrm{g} / L$ ) foi de 26,3\% (IC95\% 17,3-37,5).

Conclusões: em Teresina, a deficiência de ferro e a anemia em escolares devem ser consideradas como um problema de saúde pública que requer efetivo programa de prevenção e controle. No entanto, a anemia parece não ser explicada apenas pela deficiência de ferro; outros fatores etiológicos devem, portanto, ser considerados, a exemplo da deficiência de outros micronutrientes, infecções/infestações parasitárias, distúrbios hereditários e exposição a poluentes ambientais.

Palavras-chave Hemoglobinas, Ferritinas, Escolares, Deficiência de ferro, Anemia ferropriva 


\section{Introdução}

O indivíduo, com nutrição adequada, armazena ferro nos tecidos, que é utilizado quando a absorção desse mineral se torna insuficiente, em virtude da baixa ingestão e/ou biodisponibilidade. A quantidade de ferro armazenada, principalmente no fígado, é um marcador biológico do estado nutricional de ferro. ${ }^{1}$ A deficiência orgânica de ferro ocorre em três estágios seqüenciais: depleção das reservas, geralmente definidas por baixas concentrações de ferritina; eritropoiese deficiente em ferro, com redução do transporte do mineral e caracterizada por um aumento nas concentrações de receptores da transferrina e protoporfirina eritrocitária livre; e o terceiro estágio, que é a anemia por deficiência de ferro, que se caracteriza por um suprimento inadequado do mineral para a síntese de hemoglobina. ${ }^{2}$ Logo, estágios prévios da deficiência de ferro antecedem o aparecimento da anemia ferropênica e podem ocasionar importantes efeitos nocivos à saúde humana. ${ }^{3-5}$

A deficiência de ferro e a anemia ferropênica têm origem multicausal, cujos determinantes estão direta ou indiretamente relacionados com a ingestão e utilização biológica do mineral. Nesse sentido, o aporte dietético inadequado do ferro e/ou dos agentes facilitadores e inibidores da sua absorção, 6 bem como das infecções crônicas 7 e infestações, principalmente, das parasitoses intestinais, 8,9 são fatores de risco potencial ao status inadequado de ferro. Esses distúrbios nosológicos provocam, via de regra, anorexia, diminuição na absorção do ferro, maior perda sanguínea, destruição e menor produção de hemácias. ${ }^{1}$

Essa carência nutricional tem sido considerada como um importante problema de saúde pública, sobretudo, nos países em desenvolvimento, tanto em termos de magnitude, distribuição espacial, quanto nos seus reflexos negativos à saúde do indivíduo. $\mathrm{Na}$ infância, os efeitos deletérios mais evidentes se traduzem no retardo do desenvolvimento psicomotor, ${ }^{3}$ diminuição da capacidade de aprendizado, com perda significativa da habilidade cognitiva, 4 apatia, anorexia, déficit estatural, 5 baixo peso ao nascer e aumento da mortalidade perinatal. ${ }^{2}$

No Brasil, são raros os dados de prevalência de deficiência de ferro e anemia em escolares, a partir de estudos de base populacional.10-12 Por outro lado, a adoção de um novo ponto de corte para a definição de anemia em crianças de 5 a 11 anos $(\mathrm{Hb}<11,5$ g/dL), a partir de 1999,13 em comparação com o recomendado até $1998(\mathrm{Hb}<12,0 \mathrm{~g} / \mathrm{dL})$, tem dificultado, sobremaneira, uma análise comparativa entre estudos de prevalência realizados antes e após essa data, bem como traçar tendências seculares da ocorrência desse problema nutricional nesse grupo etário. No entanto, dados de anemia nutricional, na idade pré-escolar, têm revelado alta prevalência, com sinais evidentes de incremento significativo na sua magnitude, segundo análises de tendência temporal realizadas no município de São Paulo ${ }^{14}$ e no Estado da Paraíba. 15

Visando contribuir para uma melhor composição da cartografia da deficiência de ferro no Brasil, esse estudo se propõe a avaliar o estado nutricional de ferro, a partir das estimativas das concentrações de hemoglobina e das reservas corporais de ferro em escolares da rede pública de ensino de Teresina, Estado do Piauí.

\section{Métodos}

Estudo de corte transversal, do tipo descritivo, envolvendo crianças de 7 a 11 anos de idade, de ambos os sexos, regularmente matriculadas em escolas públicas do município de Teresina, Piauí, no período de agosto/setembro de 2000.

$\mathrm{Na}$ determinação do tamanho amostral, foi estimada uma prevalência de $10,0 \%$ de níveis de hemoglobina inferiores a 11,5 g/dL. 10 Tomou-se como base para definição da amostra, a equação descrita na fórmula16: $N=\left[E^{2} \cdot p(1-p)\right] / D$, onde $E$ é o limite de confiança $(E=1,96)$, considerando uma confiabilidade de $95 \%$ nessa estimativa populacional; $\mathrm{p}$ a prevalência estimada ( $p=10,0 \%$ ); e $\mathrm{D}$, a precisão aceita para a prevalência estimada $(D=3,2 \%)$. O tamanho amostral mínimo (N) obtido foi de 333 escolares. Uma vez que a amostra não foi aleatória simples, mas por conglomerado, levou-se em consideração o efeito do desenho (ED). No cálculo desse efeito, tomou-se como tamanho do conglomerado (m) 50 escolares e uma variabilidade interconglomerados (VIC) da ordem de 0,023, e aplicou-se a fórmula17: $\mathrm{ED}=[1+(\mathrm{m}-1) \mathrm{VIC}]$, resultando em um efeito do desenho de, aproximadamente, 2,1. Logo, o tamanho amostral foi corrigido para 700 escolares. Para compensar eventuais perdas no processo de seleção da amostra, bem como problemas de ordem técnica e operacional, corrigiu-se o tamanho amostral, com uma adição de 10,0\%, perfazendo um total de 770 escolares.

Para a seleção da amostra, procedeu-se o levantamento da população elegível, mediante a identificação de todas as escolas públicas cadastradas na Secretaria de Educação do Município, aptas para o funcionamento no ano de 2000. Na primeira etapa do 
processo de casualização, a unidade amostral foi o conglomerado (escola). Utilizando-se uma tabela de números aleatórios, foram selecionadas 15 escolas de um universo de 147 . Na segunda etapa, correspondente à seleção dos escolares, 50 em cada escola, a casualização simples também se processou mediante o uso de uma tabela de números aleatórios, gerados dentro do universo de crianças elegíveis, em cada escola selecionada na primeira etapa.

Para a determinação das concentrações de ferritina sérica, em virtude dos elevados custos operacionais para esse tipo de análise biológica, tomou-se uma sub-amostra, representando cerca de $25 \%$ dos escolares, resultando em um subgrupo de 207 escolares, escolhidos aleatoriamente.

A coleta de sangue foi realizada após jejum noturno de 12 horas, mediante punção venosa cubital, onde foram colhidos $6 \mathrm{~mL}$ de sangue, sendo $4 \mathrm{~mL}$ para análise da concentração de hemoglobina e $2 \mathrm{~mL}$ para a análise da ferritina sérica. A colheita foi realizada na própria escola, sempre no mesmo horário matinal, por um profissional de enfermagem, com larga experiência na área, sobretudo com crianças, sob a supervisão da coordenadora do estudo. As amostras foram enviadas imediatamente ao Centro de Diagnóstico Raul Bacelar, da Fundação Municipal de Saúde de Teresina/Piauí, que se responsabilizou pelas análises hematológica $\mathrm{e}$ bioquímica do estudo.

Para determinação da hemoglobina, foram necessários $4 \mathrm{~mL}$ de sangue para diluição em uma gota de anticoagulante Hemstab (EDTA $10 \mathrm{~g} / \mathrm{dl}$ ), visto que, no estudo piloto, quando utilizada quantidade menor, as amostras hemolisavam, sendo as mesmas desprezadas. A análise foi realizada, no máximo, duas horas após o recebimento pelo laboratório. Para tanto, utilizou-se o aparelho de automação completa Cell-Dyn System 3000 (Abbott Laboratories, Abbott Park, IL, USA), onde uma diluição de 1:251 da amostra é combinada com o diluente (sheat) e o reagente (hemoglobin/lyse), na câmara de homogeneização. As hemácias são lisadas e a hemoglobina é liberada. A concentração de hemoglobina foi determinada usando um método modificado de cianometahemoglobina, onde foram feitas cinco leituras separadas para cada amostra e a média foi apresentada como resultado. Para definir a presença de anemia, utilizou-se o ponto de corte $\mathrm{Hb}<$ $11,5 \mathrm{~g} / \mathrm{dL}$.

Para a determinação da ferritina sérica, adotouse o método de ensaio imunoenzimático, modificado pela Divisão Diagnóstica do Abbott Laboratories, que usa micropartículas a partir do método enzima imunoensaio (ELISA), resultando na técnica imunoensaio de enzimas em micropartículas (MEIA), que permite a realização de ensaios com maior precisão. A técnica foi realizada no equipamento System AxSYM (Abbott Laboratories, Abbott Park, IL, USA), que possui automação completa, com sistema randômico, o que possibilita análises com maior rapidez. Utilizou-se o reagente da ferritina, células matrix, controles alto, médio e baixo e calibradores da ferritina, para caracterização da depleção das reservas corporais de ferro o valor de referência FerS $<15,0 \mu \mathrm{g} / \mathrm{L}$.

$\mathrm{Na}$ análise estatística das variáveis, empregou-se o teste de Kolmogorov-Smirnov, para verificar se a distribuição gaussiana ajustava-se aos valores de hemoglobina e ferritina sérica. As concentrações de hemoglobina tiveram distribuição não normal, mesmo após transformação logarítimica $\left(\log _{10}\right)$, e foram descritas sob a forma de mediana, com intervalo interquartílico. As concentrações de ferritina sérica mostraram distribuição normal, após transformação logarítimica $\left(\log _{10}\right)$ e foram descritas pela média geométrica e intervalo de confiança. $\mathrm{Na}$ descrição das proporções, procedeu-se uma aproximação da distribuição binominal à distribuição normal pelo intervalo de confiança de 95\%. A comparação entre as proporções nos grupos independentes foi analisada pelo teste do qui-quadrado. Em todos os testes, adotou-se o nível de significância de $95 \%$, e utilizou-se o pacote estatístico SPSS for Windows, versão 13.1 (SPSS Inc., Chicago, IL, 2005).

O protocolo de pesquisa foi submetido e aprovado pelo Comitê de Ética da Universidade Federal de Pernambuco, sob o Protocolo de Pesquisa $n^{\circ}$ 129/2000-CEP/CCS, de acordo com a Resolução 196/96 do Conselho Nacional de Saúde, tendo em vista ser o projeto referente à tese de doutorado apresentada à citada universidade. Antes do início da pesquisa, foram estabelecidos contatos com a Secretaria Estadual de Educação do Piauí, a fim de se obter autorização para a realização da mesma. Após sorteio das escolas participantes, foram realizadas reuniões com diretores e professores das escolas, no sentido de apresentar a proposta de trabalho, a qual foi prontamente aceita por todos. Seguindo-se o protocolo, o projeto foi apresentado aos pais ou responsáveis pelas crianças, a fim de se obter autorização para a participação das mesmas. Todos foram esclarecidos sobre os objetivos, procedimentos e benefícios da pesquisa, inclusive sobre a livre escolha das crianças em participar ou não da pesquisa e também sobre a possibilidade de retirarse da pesquisa no momento que desejassem, sem qualquer prejuízo. 
Figura 1

Curva de distribuição das concentrações de hemoglobina em escolares do município de Teresina, Piauí, 2000.

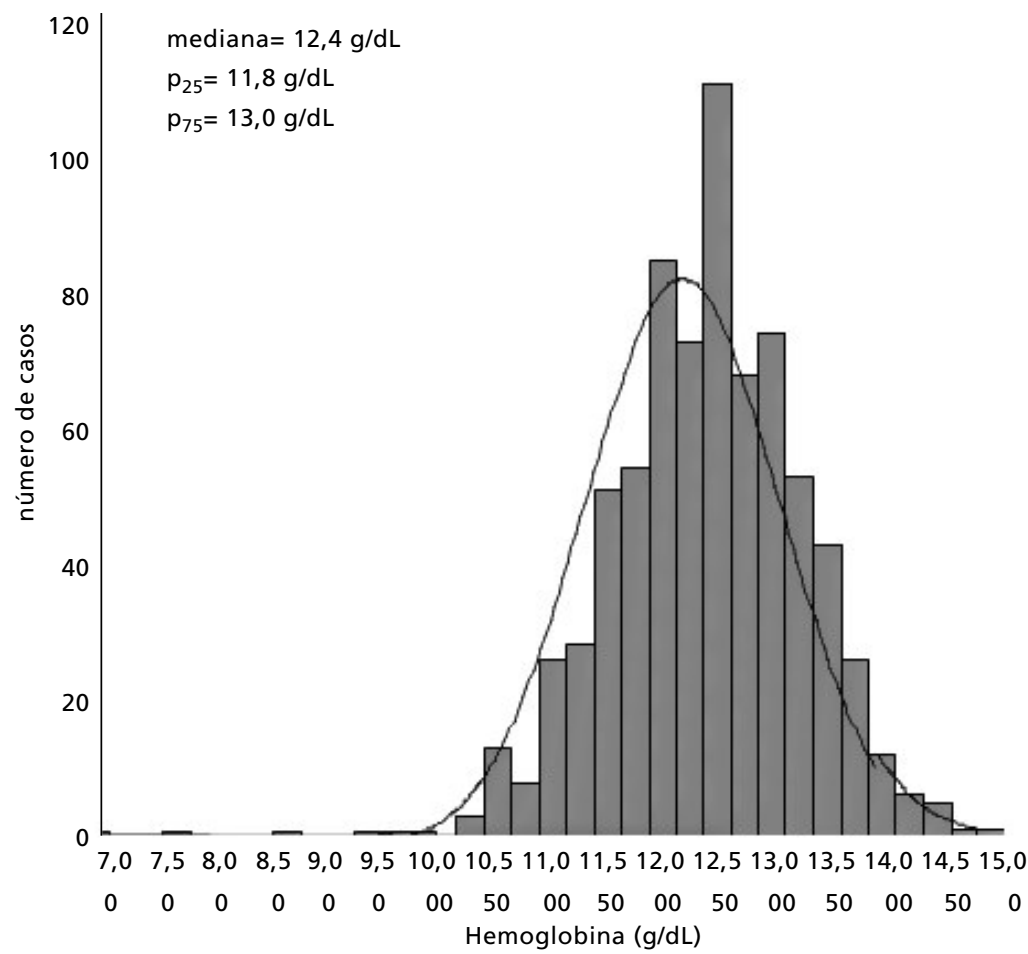

Figura 2

Curva de distribuição das concentrações de ferritina sérica em escolares do Município de Teresina, Piauí, 2000.

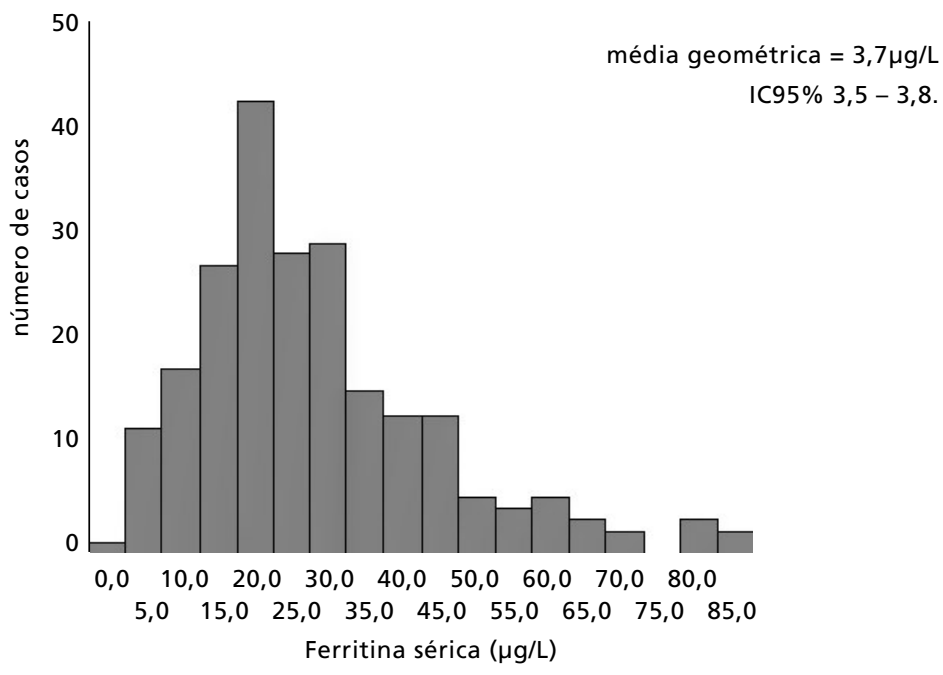

\section{Resultados}

Da amostra total estimada (770 escolares), foram analisadas concentrações de hemoglobina e ferritina sérica em 747 e 207 escolares, respectivamente. O estudo registrou 23 perdas, representando $3 \%$ da amostra estimada, sendo julgada desnecessária a sua reposição. As perdas foram em decorrência da ausência da criança na escola no dia da colheita de sangue, inconsistência nos dados de identificação, bem como alterações no material biológico, que impossibilitaram as análises bioquímicas.

Com relação ao perfil dos escolares quanto ao sexo e a idade, observou-se que $46,85 \%$ eram do sexo masculino e $53,15 \%$ do sexo feminino, cuja distribuição por faixa etária foi de $85,54 \%$ com idades de sete a nove anos e $14,46 \%$ na faixa de 9 a 12 anos.

As concentrações de hemoglobina dos escolares estudados apresentaram uma curva de distribuição não normal $(\mathrm{Z}=1,4 ; p=0,04)$, segundo o teste de Kolmogorov-Smirnov (Figura 1). Procedendo-se a transformação logarítmica $\left(\log _{10}\right)$, a variável permaneceu com distribuição não-gaussiana $(p<0,01)$, com mediana de $12,4 \mathrm{~g} / \mathrm{dL}$ e intervalo interquartílico de 1,2 $\mathrm{g} / \mathrm{dL}\left(\mathrm{P}_{25}=11,8 \mathrm{~g} / \mathrm{dL}\right.$ e $\mathrm{P}_{75}=$ $13,0 \mathrm{~g} / \mathrm{dL})$. As concentrações de ferritina sérica também não apresentaram distribuição normal $(\mathrm{Z}=1,5 ; p=0,02)$, segundo o teste de KolmogorovSmirnov (Figura. 2). No entanto, após a transformação logarítmica $\left(\log _{10}\right),(Z=1,16 ; p=0,14)$, a variável apresentou distribuição gaussiana, sendo descrita sob a forma de média geométrica $(3,7 \mu \mathrm{g} / \mathrm{L})$ e o seu respectivo intervalo de confiança $\left(\mathrm{IC}_{95 \%} 3,5\right.$ $3,8)$.

A prevalência de concentrações inadequadas de hemoglobina $(\mathrm{Hb}<11,5 \mathrm{~g} / \mathrm{dL})$ foi de $14,3 \%\left(\mathrm{IC}_{95 \%}\right.$ 12,2-17,4). O percentual de reservas corporais de ferro inadequadas $(\mathrm{FerS}<15 \mu \mathrm{g} / \mathrm{L})$ foi de $20,3 \%$ ( $\mathrm{IC}_{95 \%}$ 15,2-26-6), demonstrando que, aproximadamente, um em cada cinco escolares apresentou concentrações sugestivas de um quadro de deficiência do mineral.

A distribuição das concentrações inadequadas de hemoglobina e ferritina sérica, segundo o sexo e a faixa etária, está representada na Tabela 1. Observase que houve um comportamento similar entre as proporções de anêmicos e de escolares com baixas reservas corporais de ferro, entre os sexos e faixas etárias.

No entanto, não houve correlação $(\mathrm{r}=0,1$; $p=0,168)$ entre as concentrações de hemoglobina e 
Concentrações inadequadas de hemoglobina e ferritina sérica, segundo o sexo e a faixa etária, em escolares do município de Teresina, Piauí, 2000.

\begin{tabular}{|c|c|c|c|c|c|c|}
\hline \multirow[t]{2}{*}{ Variáveis } & \multicolumn{3}{|c|}{ Hemoglobina $(\mathrm{g} / \mathrm{dL})<11,5$} & \multicolumn{3}{|c|}{ Ferritina $(\mu \mathrm{g} / \mathrm{L})<15,0$} \\
\hline & $\mathrm{n}$ & $\%(I C) * *$ & $p^{*}$ & $\mathrm{n}$ & $\%(I C) * *$ & $p^{*}$ \\
\hline \multicolumn{7}{|l|}{ Sexo } \\
\hline Masculino $(n=350)$ & 50 & $\begin{array}{c}14,3 \\
(10,9-18,5)\end{array}$ & 0,98 & 19 & $\begin{array}{c}20,4 \\
(13,1-30,3)\end{array}$ & 0,19 \\
\hline Feminino $(n=397)$ & 57 & $\begin{array}{c}14,4 \\
(11,1-18,3)\end{array}$ & & 23 & $\begin{array}{c}21,1 \\
(14,8-31,5)\end{array}$ & \\
\hline Total $(n=747)$ & 107 & $\begin{array}{c}14,3 \\
(12,2-17,4)\end{array}$ & & 42 & $\begin{array}{c}21,3 \\
(25,3-27,8)\end{array}$ & \\
\hline \multicolumn{7}{|l|}{ Idade (anos) } \\
\hline $7+9(n=639)$ & 91 & $\begin{array}{c}14,2 \\
(11,7-17,3)\end{array}$ & 0,88 & 37 & $\begin{array}{c}21,0 \\
(15,4-27,9)\end{array}$ & 0,24 \\
\hline $9+12(n=108)$ & 16 & $\begin{array}{c}14,8 \\
(9,0-23,2)\end{array}$ & & 5 & $\begin{array}{c}16,1 \\
(6,1-34,5)\end{array}$ & \\
\hline Total $(n=747)$ & 107 & $\begin{array}{c}14,3 \\
(12,2-17,4)\end{array}$ & & 42 & $\begin{array}{c}20,3 \\
(15,2-26,6)\end{array}$ & \\
\hline
\end{tabular}

*Teste do qui-quadrado

** Prevalência + Intervalo de confiança de $95 \%$.

Figura 3

Correlação entre as concentrações de hemoglobina e ferritina sérica em escolares do Município de Teresina, Piauí, 2000.

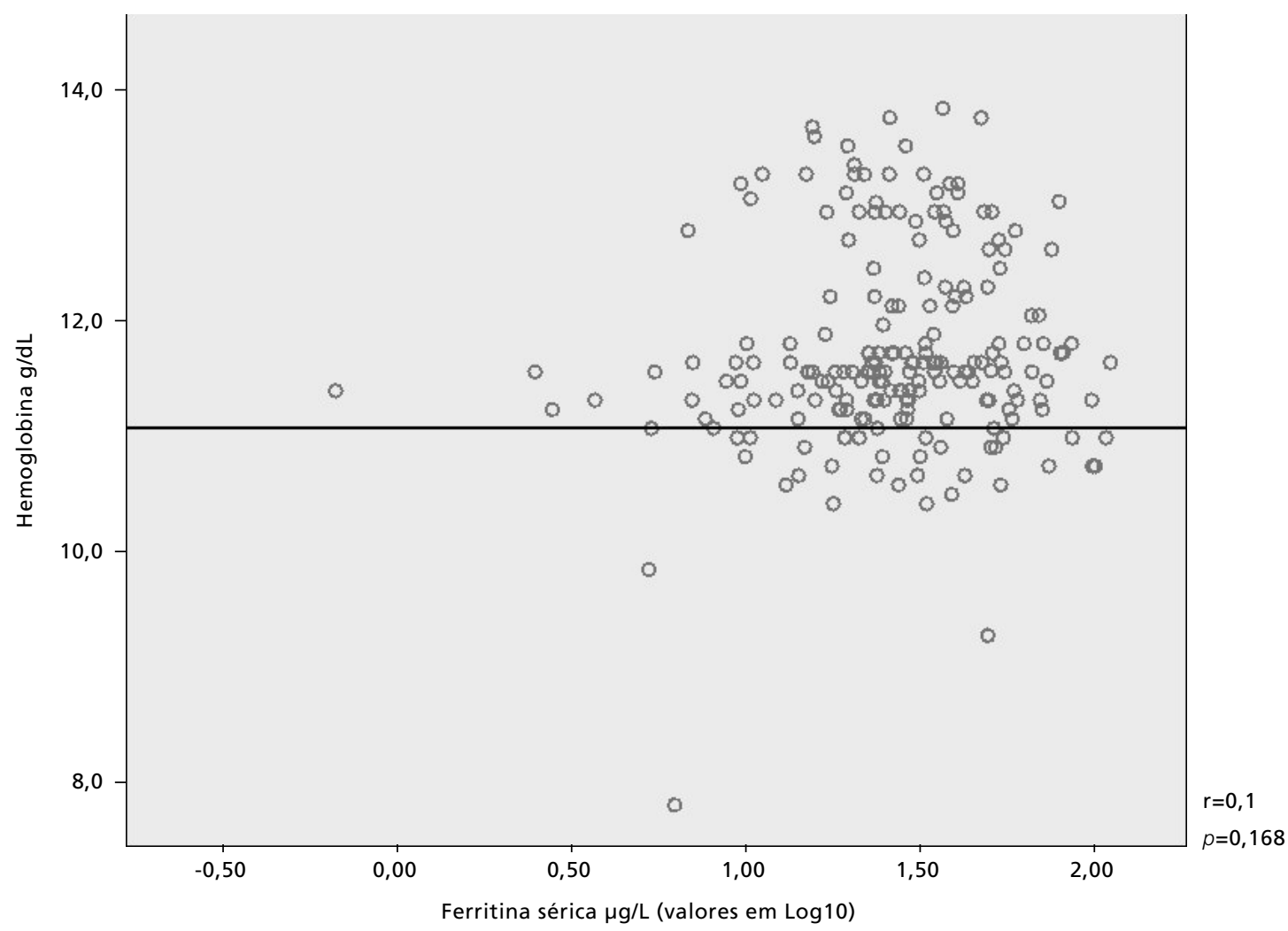


ferritina sérica (Figura 3), uma vez que apenas 9,9\% $\left(\mathrm{IC}_{95 \%}\right.$ 6,4-14,9) dos escolares apresentaram concomitantemente concentrações baixas de hemoglobina $(<11,5 \mathrm{~g} / \mathrm{dL})$ e ferritina sérica $(<15 \mu \mathrm{g} / \mathrm{L})$. Por outro lado, do total de crianças com concentrações de hemoglobina inadequadas $(n=80)$, apenas 21 também apresentaram concentrações baixas de ferritina sérica, levando-se a inferir que apenas $26,3 \%\left(\mathrm{IC}_{95 \%}\right.$ 17,3-37,5) dos casos de anemia observados nessa casuística seriam de origem ferropênica.

\section{Discussão}

A deficiência em micronutrientes, também denominada de fome oculta, tem sido considerada como um importante problema de saúde pública, em termos de magnitude, distribuição espacial e efeitos deletérios à saúde. No caso específico da carência de ferro, e da subseqüente anemia, a deficiência tem assumido caráter cosmopolita e atinge proporções extremamente elevadas, sobretudo nos grupos de maior vulnerabilidade, como é o caso das gestantes escolares e pré-escolares. 1

A prevalência de concentrações inadequadas de hemoglobina, observada nos escolares do município de Teresina, categoriza a anemia como um problema de saúde pública do tipo leve, de acordo com os critérios adotados pela WHO. 1

A confrontação dos resultados encontrados nesse estudo com aqueles divulgados por outros, reportando prevalência de anemia em escolares, 8,12 tem sido dificultada, sobremaneira, em virtude do ponto de corte até então utilizado por esses estudos $(\mathrm{Hb}<$ $12,0 \mathrm{~g} / \mathrm{dL}) .13$ No entanto, quando plausível a comparação, os nossos resultados não se distanciam daqueles registrados em outros municípios brasileiros, particularmente no Nordeste, onde foram verificadas proporções de escolares anêmicos bem próximas às encontradas em Teresina. Nesse sentido, Santos et al.,10 desenvolvendo um estudo transversal, com amostra probabilística de 426 alunos do município de Maceió, na faixa etária de seis a dez anos, encontraram prevalência de $10,0 \%$ de anemia. Por sua vez, Pereira et al. 18 observaram, em escolares da zona da mata de Pernambuco, concentrações médias de hemoglobina em torno de $11,9 \mathrm{~g} / \mathrm{dL}$ e, portanto, muito similares às nossa observações.

Entretanto, em Minas Gerais, Norton et al. 19 encontraram uma média das concentrações de hemoglobina da ordem de 12,8 g/dL. Na cidade de Campinas, São Paulo, Moura et. al.11 registraram prevalência média de $12,4 \%$ de anemia em escolares, sendo que os pontos de cortes para caracterização da anemia foram diferentes para as idades de cinco a oito anos incompletos $(\mathrm{Hb}<11,5 \mathrm{~g} / \mathrm{dl})$ e de oito a menores de doze anos $(\mathrm{Hb}<11,9 \mathrm{~g} / \mathrm{dL})$. Prevalências de anemia mais elevadas foram verificadas por Sichieri et al., 6 alcançando 25,7\% $(\mathrm{Hb}<11,5 \mathrm{~g} / \mathrm{dL})$ em escolares procedentes de famílias de baixa renda de uma comunidade rural do Paraná. Esses resultados poderiam ser atribuídos ao habitat extremamente desfavorável que caracteriza a maioria dos espaços rurais no Brasil.

Recentemente, três estudos desenvolvidos em diferentes regiões do Brasil apontam diferenças e similaridade em relação aos resultados aqui encontrados. Na cidade de São Paulo, Santos et al., ${ }^{20}$ utilizando ponto de corte $\mathrm{Hb}<11,5 \mathrm{~g} / \mathrm{dL}$, encontraram prevalência de $24,4 \%$ em escolares de favelas. Por sua vez, Heijblom et al.,21 objetivando avaliar a tendência temporal da anemia em escolares da primeira série do ensino fundamental da rede pública de educação de Brasília, observaram uma estabilidade na ocorrência da anemia, com cifras de $11,9 \%$ para o ano de 1998 e de $12,5 \%$ para o ano de 2004. No entanto, estudo desenvolvido por Pereira et al. 18 mostrou prevalência extremamente elevada de anemia $(53,2 \%)$ em escolares do município de São João, zona da mata sul do Estado de Pernambuco, embora apenas $11,2 \%$ dos casos de anemia tivessem microcitose.

A prevalência de concentrações inadequadas de ferritina sérica, nos escolares de Teresina (20,3\%), foi semelhante àquela encontrada na cidade de Campinas, Estado de São Paulo (19,3\%).11 Isso vem demonstrar que a ocorrência de taxas elevadas de reservas corporais inadequadas de ferro, bem como da anemia, são reflexos importantes da carência de ferro, mesmo entre escolares que habitam contextos geográficos diferenciados.

Vale ressaltar que $63,0 \%\left(\mathrm{IC}_{95 \%}: 56,1-69,5\right)$ dos escolares apresentaram concentrações de ferritina sérica inferiores a $30 \mu \mathrm{g} / \mathrm{L}$, valor do ponto de corte recomendado para a estimativa de depleção das reservas de ferro na presença de infecção. ${ }^{1}$ Considerando-se que a população estudada vive em um ecossistema extremamente propício às infecções, sobretudo subclínicas, ${ }^{9,22}$ corre-se o risco de subestimar a prevalência das reservas inadequadas de ferro, no contexto das escolas de Teresina, adotandose, exclusivamente, o ponto de corte de $15 \mu \mathrm{g} / \mathrm{L}$, para a estimativa da carência marcial.

A distribuição homogênea da depleção das reservas corporais de ferro e da anemia, nos dois sexos, nos escolares do município de Teresina difere 
da literatura disponível, uma vez que tem sido reportada uma maior susceptibilidade das crianças do sexo masculino à carência em ferro, 7,15 bem como a outros micronutrientes, 5 a exemplo da vitamina A. Diniz e Santos 23 observaram que crianças do sexo masculino têm um risco relativo duas vezes maior de apresentar xeroftalmia moderada e três vezes maior de apresentar xeroftalmia grave, quando comparada com crianças do sexo feminino. Dados oriundos de seis países da África (Gana, Quênia, Malaui, Moçambique e Tanzânia) e de dois países da Ásia (Indonésia e Vietnan), mostraram também maior vulnerabilidade de escolares do sexo masculino à deficiência de ferro e à anemia ferropênica. ${ }^{24}$

Considerando o grupo de escolares pesquisados, os resultados relativos à distribuição das reservas corporais de ferro e da anemia são concordantes com a literatura que aponta maior vulnerabilidade em crianças mais jovens. 15 Discordante desse estudo, em pesquisa realizada com escolares do Kazakhstan, 25 não foi verificada a correlação entre a ocorrência de anemia e idade da criança. Dessa forma, as informações disponíveis na literatura especializada 7,8,26,27 ainda não permitem uma síntese conclusiva sobre a tendência da carência de ferro e anemia com relação à progressão etária, em crianças na idade escolar.

A maior prevalência da hipoferritinemia $(20,3 \%)$, em relação àquela observada para a anemia (14,3\%), é um achado consistente, se analisado na perspectiva do paradigma da história natural da carência, onde ocorreria inicialmente a diminuição das reservas corporais do ferro, e a redução da hemoglobinogênese se instalaria apenas em um estágio mais tardio, com a acentuada depleção dos estoques do mineral. No entanto, a ausência de correlação entre as concentrações de hemoglobina e de ferritina sérica, bem como a ocorrência de anemia ferropênica $(\mathrm{Hb}<$ $11,5 \mathrm{~g} / \mathrm{dL}$ e FerS $<15,0 \mu \mathrm{g} / \mathrm{L}$ ) em $26,3 \%$ dos escolares são achados que merecem reflexão. Concentrações de hemoglobina têm sido usadas isoladamente como indicador da anemia por defi-

\section{Referências}

1. WHO (World Health Organization). Iron deficiency anaemia - assessment, prevention, and control: a guide for programme managers. Washington, DC; 2001.

2. DeMaeyer EM, Dallman P, Gurney JM. Preventing and controlling iron deficiency anemia through primary health care. Geneva: World Health Organization; 1989.

3. Walter T, De Andraca I, Chadud P, Perales CG. Iron deficiency anemia: adverse effects on infants psychomotor development. Pediatrics. 1989; 84: 7-17. ciência de ferro, na maioria dos países em desenvolvimento, uma vez que tem sido aceito que a anemia em escolares e adolescentes é decorrente, principalmente, dessa deficiência ${ }^{7,28}$ e outros testes confirmatórios geralmente não estão disponíveis, em virtude de dificuldades técnicas ou custos elevados. ${ }^{1}$ Vale ressaltar que, essa majoritária etiologia ferropênica da anemia não tem sido respaldada de forma consensual pela literatura especializada, a partir de estudos epidemiológicos onde foram usados outros indicadores para caracterização da anemia. ${ }^{25-}$ 27,29

Nesse sentido, a anemia observada nos escolares de Teresina parece não ser explicada apenas pela deficiência de ferro, outros fatores etiológicos devem ser igualmente considerados, a exemplo da deficiência por infecções, 25,29 outros micronutrientes, 30 infestações parasitárias, 7,22 distúrbios hereditários,26,27 exposição a poluentes ambientais, 31 entre outros.

Sumariando, a deficiência de ferro e a anemia em escolares do município de Teresina devem ser consideradas um problema nutricional importante, que requer medidas efetivas de prevenção e controle. Espera-se que políticas públicas voltadas para o combate a essa carência nutricional, a exemplo do programa de fortificação das farinhas de trigo e milho, com ferro e ácido fólico, 32 implantado em todo território brasileiro, tenham o impacto desejável no enfrentamento desse problema nutricional, sobretudo em contextos ecológicos de grande vulnerabilidade à carência marcial, como é o caso da região Nordeste do Brasil e outros bolsões de pobreza do país.

\section{Agradecimentos}

Agradecemos à Coordenação de Aperfeiçoamento de Pessoal de Ensino Superior (CAPES) do Ministério da Educação e à Fundação Municipal de Saúde de Teresina, pelo suporte técnico e logístico.
4. Halterman JS, Kaczorowski JM, Aligne CA, Auinger P, Szilagyi PG. Iron deficiency and cognitive achievement among school-aged children and adolescents in the United States. Pediatrics. 2001; 107: 1381-6.

5. Rivera JA, Hotz C, Gonzalez-Cossio T, Neufeld L, GarciaGuerra A. The effect of micronutrient deficiencies on child growth: a review of results from community-based supplementation trials. J Nutr. 2003; 133 (11 Suppl 2): 4010-20. 
6. Sichieri R, Mathias T, Moura AS. Stunting, high weight-forheight, anemia e dietary intake among Brazilian students from a rural community. Nutr Res. 1996; 16: 201-9.

7. Stoltzfus RJ, Chwaya HM, Tielsch JM, Schulze KJ, Savioli L. Epidemiology of iron deficiency anemia in Zanzibari schoolchildren: the importance of hookworms. Am J Clin Nutr. 1997; 65: 153-9.

8. Tsuyuoka R, Bailey JW, Guimarães AMAN, Gurgel RQ, Cuevas LE. Anemia and intestinal parasitic infections in primary school students in Aracaju, Sergipe, Brazil. Cad Saúde Pública. 1999; 15: 413-21.

9. Brito LL, Barreto ML, Silva RDCR, Assis AMO, Reis MG, Parraga I, Blanton RE. Fatores de risco para anemia por deficiência de ferro em crianças e adolescentes parasitados por helmintos intestinais. Rev Panam Salud Publica. 2003; 14: 422-31

10. Santos CD, Santos LMP, Figueiroa JN, Marroquim PMG, Oliveira MAA. Anemia em escolares da $1^{\text {a }}$ série do ensino fundamental da rede pública de Maceió - AL. Cad. Saúde Pública. 2002; 18: 1757-63.

11. Moura EC, Santos AM, Pacheco CE. Anemia ferropriva em escolares de Campinas, São Paulo: prevalência, sensibilidade e especificidade de testes laboratoriais. Rev Bras Saúde Matern Infant. 2001; 1: 123-27.

12. Stefanini MLR, Colli C, Lerner BR, Lei DLM, Chaves SP, Pietro MSDi, Oliveira AAM, Szarfarc SC. Anemia and malnutrition in children at public schools in Osasco, São Paulo (Brasil). Cad. Saúde Pública. 1995; 11: 439-47.

13. UNICEF (United Nations Children's Fund), UNU (United Nations University), WHO (World Health Organization), MI (Micronutrient Iniciative). Preventing iron deficiency in women and children: technical workshop. Boston: International Nutrition Foundation; 1999.

14. Monteiro CA, Szarfarc SC, Mondini L. Tendência secular da anemia na infância na cidade de São Paulo (1984-1996). Rev Saúde Pública. 2000; 34 (Supl 6): 62-72.

15. Oliveira RS, Diniz AS, Benigna MJC, Miranda-Silva SM, Lola MM, Gonçalves MC, Asciutti-Moura LS, Rivera MA, Santos LMP. Magnitude, distribuição espacial e tendência temporal da anemia em pré-escolares do Estado da Paraíba. Rev Saúde Pública. 2002; 36: 26-32.

16. Henderson RH, Sundaresan T. Clustersampling to assess immunization coverage: a review of experience with a sampling method. Bull World Health Organ. 1982; 60: 25360 .

17. Bland M. An introduction to medical statistics. 3.ed. New York: Oxford University Press; 2004.

18. Pereira RC, Ferreira LOC, Diniz AS, Batista-Filho M, Figuerôa JN. Eficácia da suplementação de ferro combinada ou não à vitamina A no controle da anemia em escolares. Cad Saúde Pública. 2007; 23: 1415-1421.

19. Norton RC, Figueiredo RC, Diamante R, Goulart EM, Mota JA, Viana MB, Penna FJ, Leão E. Prevalence of anemia among school-children from Rio Acima (State of Minas Gerais, Brazil): use of the standardized prevalence method and evaluation of iron deficiency. Braz J Med Biol Res. 1996; 29: 1617-24.

Recebido em 31 de janeiro de 2007

Versão final apresentada em 16 de setembro de 2008

Aprovado em 30 de outubro de 2008
20. Santos EB, Amancion OMS, Oliveira CAG. Estado nutricional, ferro, cobre e zinco em escolares de favelas da cidade de São Paulo. Rev Assoc Med Bras. 2007; 53: $323-$ 8 .

21. Heijblom GS, Santos LMP. Anemia ferropriva em escolares da primeira série do ensino fundamental da rede pública de educação de uma região de Brasília, DF. Rev Bras Epidemiol. 2007; 10: 258-66.

22. Ferreira MR, Souza W, Perez EP, Lapa T, Carvalho AB, Furtado A, Coutinho HB, Wakelin D. Intestinal helminthiasis and anaemia in youngsters from Matriz da Luz, district of São Lourenço da Mata, State of Pernambuco, Brazil. Mem Inst Oswaldo Cruz. 1998; 93: 289-93.

23. Diniz AS, Santos LMP. Hipovitaminose A e xeroftalmia. J Pediatr (Rio J). 2000; 76 (supl. 3): 311-22.

24. Hall A, Bobrow E, Brooker S, Nokes MJ, Lambo J, Guyatt H, Bundy D, Adjei S, Wen ST Satoto, Subagio $H$, Rafiluuddin MZ, Miguel T, Moulin S, De Grafo Jonson J, Mukala M, Roschnik N, Sacko M, Zacher A, Mahumane B, Kihamia C, Mwanri L, Tatala S. Lwambo N, Siza J, Khanh LN, Khoi HH, Toan ND. Anaemia in schoolchildren in eight countries in Africa and Asia. Public Health Nutrit. 2001; 4: 749-56.

25. Hashizume M, Kunii O, Sasaki S, Shimoda TWakai S, Mazhitova Z, Dauletbaev D, Caypil W, Aldiyarova M, Farmer A, Yashiro Y, Chiba M. Anemia and iron deficiency among schoolchildren in the Aral Sea region, Kazakhstan. J Trop Pediatr. 2003; 49: 172-7.

26. Thurlow RA, Winichagoon $P$, Pongcharoen $T$, Gowachiparant S, Boonpradern A, Manger MS, Bailey KB, Wasantwisut E, Gibson RS. Risk of zinc, iodine and other micronutrient deficiencies among school children in North East Thailand. Eur J Clin Nutr. 2006; 60: 623-32.

27. Linpisarn S, Tienboon $P$, Promtet N, Putsyainut $P$, Santawanpat S, Fuchs GJ. Iron deficiency anemia in children with a high prevalence of haemoglobinopathies: implication for screening. Int J Epidemiol. 1996; 25: 1262-6.

28. Foo LH, Khor GL, Tee ES, Prabakaran D. Iron status and dietary intake of adolescents from a rural community in Sabah, Malaysia. Asia Pac J Clin Nutr. 2004; 13: 48-55.

29. Ahmed F, Khan MR, Islam M, Kabir I, Fuchs GJ. Anaemia and iron deficieny among adolescent schoolgirls in periurban Bangladesh. Eur J Clin Nutr. 2000; 54: 678-83.

30. Fernando SD, Paranavitane SR, Rajakaruna J, Weerasinghe $\mathrm{S}$, Silva D, Wickremasinghe AR. The health and nutritional status of school children in two rural communities in Sri Lanka. Trop Med Int Health. 2000; 5: 450-2.

31. Rondó PHC, Carvalho MSH, Souza MC, Moraes F. Lead, hemoglobin, zinc protoporphyrin and ferritin concentrations in children. Rev Saúde Pública. 2000; 40: 71-6.

32. Brasil. Ministério da Saúde. 2002. Consulta Pública n. 51 de 10 de junho de 2002. Regulamento técnico para fortificação com ferro e ácido fólico nas farinhas de trigo e nos derivados do milho. 2002. [2002 Jun 10]. Disponível em http:// www.anvisa.gov.br/consulta/2002/51_2002.htm 